\title{
Downregulation of $\beta$-catenin and Akt signaling is responsible for poor proliferation of the late passage of bone marrow mesenchymal stem cells
}

\author{
JUNFANG WU ${ }^{1}$, JIE NIU ${ }^{1}$, XIAOPENG LI ${ }^{2}$, TONGMING REN ${ }^{3}$ and FENXI ZHANG ${ }^{3,4}$ \\ ${ }^{1}$ Laboratory of Morphology, School of Basic Medical Sciences; ${ }^{2}$ Department of Ophthalmology, \\ The Third Affiliated Hospital; ${ }^{3}$ Department of Anatomy; ${ }^{4}$ Stem Cell and Biotherapy Technology \\ Research Center, Xinxiang Medical University, Xinxiang, Henan 453003, P.R. China
}

Received September 22, 2013; Accepted April 10, 2014

DOI: $10.3892 / \mathrm{mmr} .2014 .2286$

\begin{abstract}
It is well established that mesenchymal stem cells (MSCs) will partially lose their proliferative ability with continuous expansion. However, the specific mechanisms underlying this effect remain unclear. In the present study, it was identified that $\beta$-catenin was downregulated in the late passage (passage 8 ) of bone marrow mesenchymal stem cells (bmMSCs). Following $\beta$-catenin expression, the expression of phospho-Akt was also significantly decreased in the late passage of bmMSCs. More notably, overexpression of $\beta$-catenin in passage 8 of bmMSCs by transfection with pMXs- $\beta$-catenin plasmids, significantly increased cell proliferation and Akt expression. These results indicate that the downregulation of $\beta$-catenin and Akt signaling may be a critical factor for the proliferation of the late passage of bmMSCs.
\end{abstract}

\section{Introduction}

Bone marrow mesenchymal stem cells (bmMSCs) are a type of multipotent stem cells that are capable of differentiating into multiple cell types, including osteoblasts, chondrocytes, adipocytes, cardiomyocytes and neurons $(1,2)$. Currently, bmMSCs are widely utilized in regenerative medicine. The proliferative ability of bmMSCs is the most important determinant for the efficiency of bmMSC-based transplantation therapy $(1,3)$. Previous studies have demonstrated that bmMSCs may lose their proliferative ability with consecutive expansion (4). However, why the late passages of bmMSCs have low proliferative ability remains unclear.

Correspondence to: Dr Fenxi Zhang, Department of Anatomy, Xinxiang Medical University, 601 Juishui Road, Xinxiang, Henan 453003, P.R China

E-mail: fxzhang0824@gmail.com

Key words: bone marrow mesenchymal stem cells, $\beta$-catenin, Akt, cell proliferation
The Wnt/ $\beta$-catenin signaling pathway has a critical role in cell proliferation. The upregulation of $\mathrm{Wnt} / \beta$-catenin signals stimulates cell proliferation and improves cell survival $(5,6)$. Akt is also an important regulator for cell proliferation and survival (7). Previous studies have demonstrated that downregulation and deficiency of Akt impairs cell proliferation $(8,9)$. In the preliminary study, we observed that $\beta$-catenin and Akt were markedly downregulated in the late (8th) passage of bmMSCs. Therefore, it was hypothesized that the downregulation of $\beta$-catenin and Akt signals may be important in regulating the proliferative ability of the late passage of bmMSCs and the present study was designed to address this hypothesis.

\section{Materials and methods}

Materials and reagents. Dulbecco's Modified Eagle Medium (DMEM), Lipofectamine ${ }^{\circledR} 2000$, DNase I, RNeasy Mini kit and SuperScript II First Strand DNA Synthesis kit were purchased from Invitrogen Life Technologies (Carlsbad, CA, USA) and the 2X PCR Reaction Mix was obtained from Sigma-Aldrich (St. Louis, MO, USA). HyClone fetal bovine serum (FBS) and ECL western blotting substrate was purchased from Thermo Fisher Scientific Inc. (Cleveland, $\mathrm{OH}$, USA). Rabbit anti-mouse $\beta$-catenin antibody was purchased from Santa Cruz Biotechnology, Inc. (Santa Cruz, CA, USA). Phospho-Akt and $\beta$-actin primary antibodies, as well as HRP-conjugated goat anti-rabbit secondary antibody were obtained from Abcam (Cambridge, MA, USA). Precision Plus Protein prestained standards were purchased from Bio-Rad (Hercules, CA, USA). The PVDF membrane was obtained from GE Healthcare (Pittsburgh, PA, USA).

Cell culture and study protocol. BmMSCs were obtained and cultured as previously described $(1,10)$. Passage 2 and passage 8 of cells were used in the experiments. The present study was approved by the Ethics Committee of Xinxiang Medical University (Xinxiang, China).

Cell counting. The passage 2 and passage 8 of bmMSCs were plated in 24-well plates (4 wells/group). Cells were collected on days $2,4,6,8,10$ and 12 , and counted under a microscope 
(Olympus, Tokyo, Japan). The cell growth curve was drawn according to the average cell number of each group.

Western blot analysis. Western blotting was performed following the standard procedure. Proteins were extracted from passage 2 and passage 8 of bmMSCs and separated by sodium dodecyl sulfate-polyacrylamide gel (SDS-PAGE) electrophoresis. Following the electrophoresis, proteins were transferred to the polyvinylidene difluoride (PVDF) membranes. The membranes were blocked with $5 \%$ milk or $5 \%$ bovine serum albumin (BSA) in Tris-buffered saline with Tween-20 (TBS-T), and then incubated with $\beta$-catenin or phospho-Akt primary antibodies at $4^{\circ} \mathrm{C}$ overnight. Following three times washing with TBS-T, the blots were incubated with $\beta$-actin antibody at room temperature for $1 \mathrm{~h}$. Then, the blots were washed with TBS-T and incubated with HRP-conjugated secondary antibody at room temperature for $1 \mathrm{~h}$. The immunoreactive bands were visualized by enhanced chemiluminescence, and the images were scanned using a Gel Imaging Analysis System (Bio-Rad, Hercules, CA, USA).

Reverse transcription polymerase chain reaction (RT-PCR) assay. In the present study, expression of Aktl in passage 8 of bmMSCs was measured using RT-PCR assay following transfection of the cells with pMXs- $\beta$-catenin plasmids. The procedures of RNA extraction and RT-PCR reaction were conducted as previously described (10). The primers used were 5'-GTCTCTAGGGTCCAGGGCCAAAGTC-3'(Akt1 forward) and 5'-CATCTAAAAGGACAAGTGCTAGGAG-3' (Akt1 reverse); 5'-TTCTTTGCAGCTCCTTCGTTGCCG-3' ( $\beta$-actin forward) and 5'-TGGATGGCTACGTACATGGCTGGG-3' ( $\beta$-actin reverse).

$\beta$-catenin overexpression in passage 8 bmMSCs. Passage 8 bmMSCs were plated in 6-well plates. As the cells reached $80 \%$ confluency, they were transfected with pMXs- $\beta$-catenin plasmids using Lipofectamine ${ }^{\circledR} 2000$. The cells transfected with empty pMXs plasmids served as controls.

Statistical analysis. Statistical analysis was performed with SPSS 11.5 software (SPSS, Inc., Chicago, IL, USA). Data are presented as the mean \pm SD from four independent experiments. Univariate comparisons of the means were evaluated using the Student's t-test and/or one-way ANOVA with Tukey's post-hoc adjustment for multiple comparisons when appropriate. $\mathrm{P}<0.05$ was considered to indicate a statistically significant difference.

\section{Results}

Proliferation of passage 2 and passage 8 bmMSCs. As illustrated in Fig. 1, the cell growth curve revealed that the cell numbers of passage $8 \mathrm{bmMSCs}$ following plating for 2, 4, 6, 8,10 and 12 days was significantly lower than the numbers of passage 2 cells $(\mathrm{P}<0.05)$. This demonstrates that the late passage of bmMSCs have a lower proliferative ability than the early passage of cells.

Expression of $\beta$-catenin and Akt in passage 2 and passage 8 bmMSCs. Western blotting analysis demonstrated that

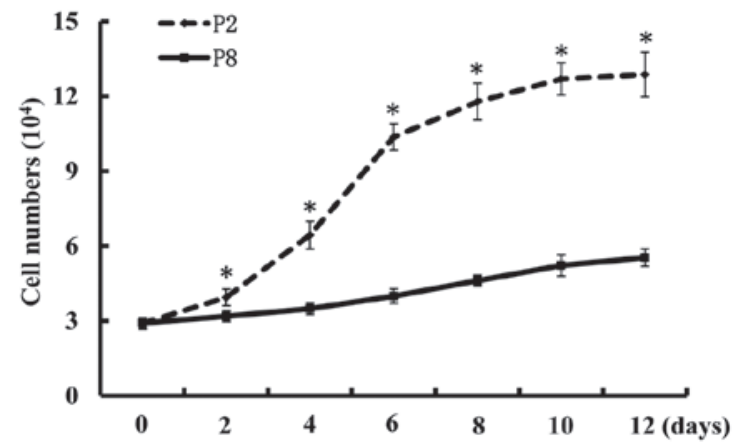

Figure 1. Growth curve illustrates the proliferation of P2 and P8 bmMSCs. Error bars represent the mean \pm SD (four independent experiments/group). ${ }^{*} \mathrm{P}<0.05$ vs. passage 8 cells. bmMSCs, bone marrow mesenchymal stem cells; $\mathrm{P}$, passage.
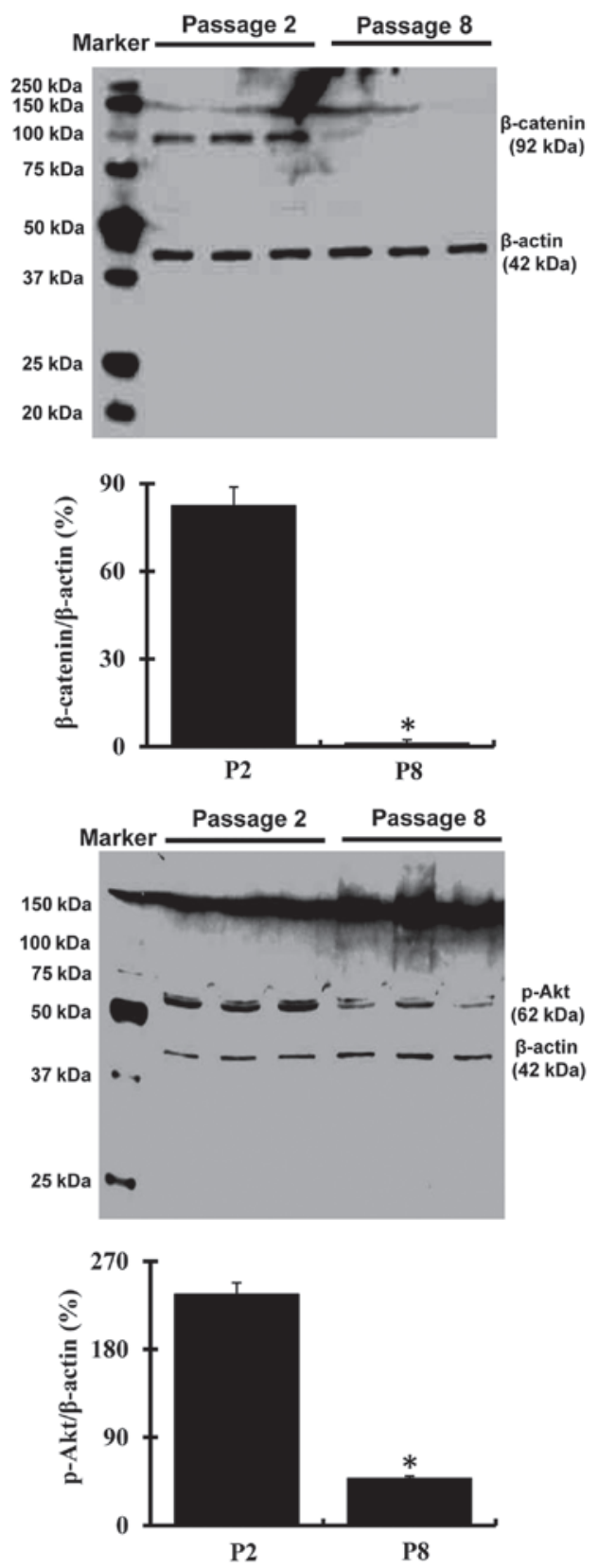

Figure 2. Expression of $\beta$-catenin and phospho-Akt in passage 2 and passage 8 bmMSCs. Bar graphs represent the mean \pm SD (four independent experiments/group). ${ }^{*} \mathrm{P}<0.05$ vs. passage 2 cells. bmMSCs, bone marrow mesenchymal stem cells. 

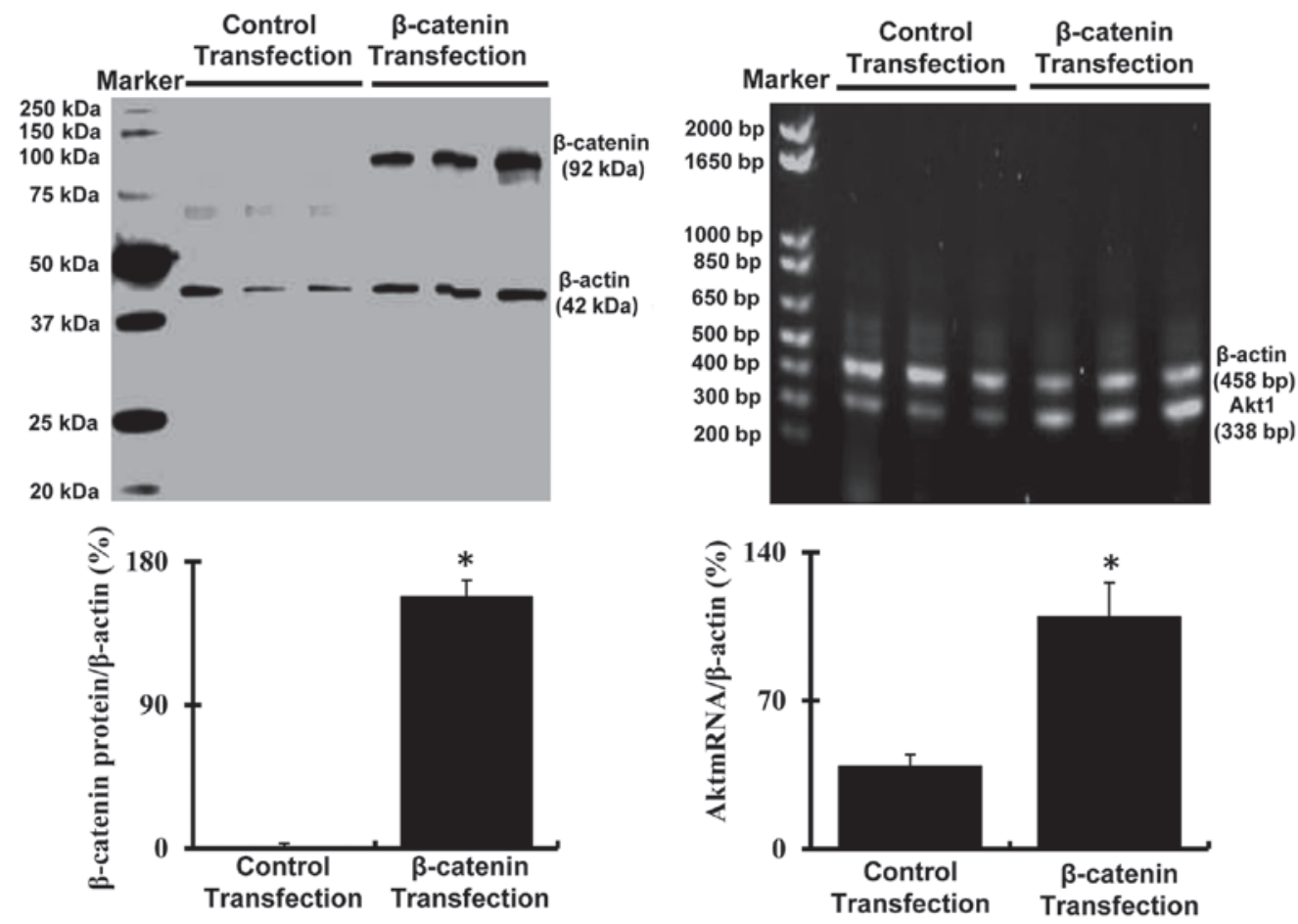

Figure 3. Western blot analysis and RT-PCR assays demonstrate $\beta$-catenin protein and Akt1 mRNA expression in passage 8 bmMSCs following transfection with empty pMXs plasmids and pMXs- $\beta$-catenin plasmids. Bar graphs represent the mean $\pm \mathrm{SD}$ (four independent experiments/group). ${ }^{*} \mathrm{P}<0.05$ vs. transfection with empty plasmids. bmMSCs, bone marrow mesenchymal stem cells; RT-PCR, reverse transcription-polymerase chain reaction.

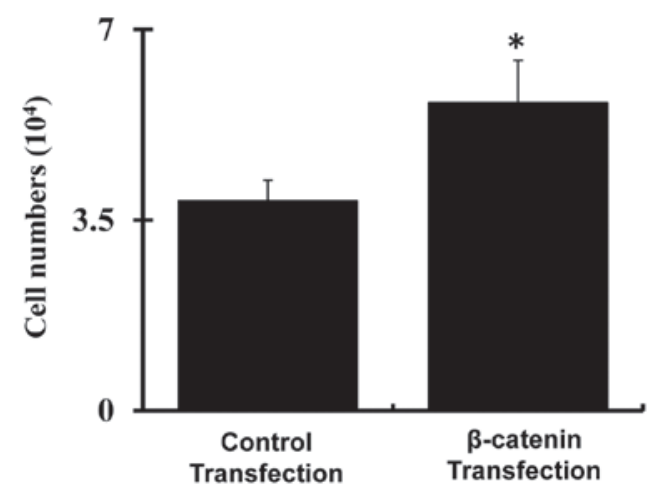

Figure 4. Cell proliferation following transfection with empty pMXs plasmids and pMXs- $\beta$-catenin plasmids for $24 \mathrm{~h}$. Bar graphs represent the mean \pm SD (four independent experiments/group). ${ }^{*} \mathrm{P}<0.05$ vs. transfection with empty plasmids.

expression of $\beta$-catenin and phospho-Akt was markedly decreased $(\mathrm{P}<0.05)$ in passage $8 \mathrm{bmMSCs}$ as compared with passage 2 cells (Fig. 2).

Expression of $\beta$-catenin and Akt in passage 8 bmMSCs following transfection with pMXs- $\beta$-catenin plasmids. As illustrated in Fig. 3, the western blot analysis revealed that the expression of $\beta$-catenin was markedly increased $(\mathrm{P}<0.05)$ in passage $8 \mathrm{bmMSCs}$ transfected with pMXs- $\beta$-catenin plasmids as compared with the cells transfected with empty pMXs plasmids. More notably, another cell proliferation signal Akt (Aktl mRNA) was also markedly upregulated $(\mathrm{P}<0.05)$ in passage 8 bmMSCs transfected with pMXs- $\beta$ catenin plasmids.
Cell proliferation following transfection of $p M X s-\beta$-catenin plasmids. As illustrated in Fig. 4, cell counting demonstrated that proliferation of passage $8 \mathrm{bmMSCs}$ was significantly increased following transfection with pMXs- $\beta$-catenin plasmids as compared with the cells transfected with empty pMXs plasmids $(\mathrm{P}<0.05)$.

\section{Discussion}

In the present study, it was demonstrated for the first time, to the best of our knowledge, that $\beta$-catenin and Akt expression are involved in the weak proliferative ability of the late passage of bmMSCs. The results revealed that $\beta$-catenin and Akt were markedly downregulated in the late passage (passage 8 ) of bmMSCs as compared with the early passage (passage 2). Overexpression of $\beta$-catenin in the late passage of bmMSCs increased their proliferation and Akt expression.

bmMSCs are the promising source of seed cells for stem cell transplant therapy. The proliferative ability of bmMSCs is the most important determinant of bmMSC-based therapeutic efficiency. Previous studies have demonstrated that the late passages of MSCs will partially lose their proliferative ability (4). As is consistent with previous studies, we also observed that the proliferation of the late passage (passage 8) of bmMSCs was markedly lower than the early passage (passage 2) cells.

More importantly, we identified that the expression of $\beta$-catenin was notably downregulated in the late passage of bmMSCs. $\beta$-catenin is a dual function protein that mainly regulates cell-cell adhesion and gene transcription. The expression of Wnt/ $\beta$-catenin signals has also been implicated in the proliferation of numerous cell types, including MSCs $(11,12)$. 
It has been demonstrated that stimulation of $\mathrm{Wnt} / \beta$-catenin signaling with its agonists promotes proliferation of MSCs (13). Downregulation or deficiency of $\beta$-catenin was also identified to inhibit the proliferative ability of other cell types (14). In the present study, we hypothesized that the downregulation of $\beta$-catenin may be responsible for the weak proliferation of the late passage of bmMSCs. Our further experiments confirmed this, which suggests that overexpression of $\beta$-catenin in the late passage of bmMSCs enhances their proliferative ability.

Akt is a serine/threonine-specific protein kinase that is important in numerous cell physiological processes, including cell metabolism, proliferation, migration, autophagy and apoptosis $(7,15,16)$. Akt is known as a cell survival signal and activation of the PI3K/Akt pathway has been observed to promote proliferation of bmMSCs (17). In the present study, we identified that phospho-Akt expression was significantly decreased in the late passage of bmMSCs following the expression of $\beta$-catenin. Previous studies have demonstrated that Akt regulates expression of $\beta$-catenin and the $\beta$-catenin signaling also participates in the regulation of Akt expression (18-20). Furthermore, it appears $\beta$-catenin regulates cell proliferation via activation of the PI3K/Akt pathway (21). In the present study, overexpression of $\beta$-catenin in the late passage of bmMSCs also enhanced Aktl expression.

In conclusion, these data revealed that $\beta$-catenin and $\mathrm{Akt}$ were markedly downregulated in passage $8 \mathrm{bmMSCs}$ and that the proliferative ability of passage $8 \mathrm{bmMSCs}$ was significantly lower than passage 2 bmMSCs. Transfection of $\beta$-catenin cDNA in passage $8 \mathrm{bmMSCs}$ enhanced the proliferation of these cells and increased Akt expression. These results indicate that $\mathrm{Wnt} / \beta$-catenin and Akt signals have an important role in the regulation of bmMSC growth.

\section{Acknowledgements}

This study was supported by a grant from the National Natural Science Foundation of China (no. 81370428).

\section{References}

1. Zhang F, Wang C, Jing S, et al: Lectin-like oxidized LDL receptor expresses in mouse bone marrow-derived mesenchymal stem cells and stimulates their proliferation. Exp Cell Res 319: 1054-1059, 2013.

2. Ferroni L, Gardin C, Tocco I, et al. Potential for neural differentiation of mesenchymal stem cells. Adv Biochem Eng Biotechnol 129: 89-115, 2013.

3. Meligy FY, Shigemura K, Behnsawy HM, et al: The efficiency of in vitro isolation and myogenic differentiation of MSCs derived from adipose connective tissue, bone marrow, and skeletal muscle tissue. In Vitro Cell Dev Biol Anim 48: 203-215, 2012.
4. Boroujeni ME, Gowda P, Johnson J, et al: The proliferation and differentiation capacity of bone marrow derived-human mesenchymal stem cells in early and late doubling. Asian J Biochem 7: 27-36, 2012.

5. Masckauchán TN, Shawber CJ, Funahashi Y, et al: Wnt/beta-catenin signaling induces proliferation, survival and interleukin-8 in human endothelial cells. Angiogenesis 8: 43-51, 2005.

6. Sarkar S, Swiercz R, Kantara C, et al: Annexin A2 mediates up-regulation of NF- $x \mathrm{~B}, \beta$-catenin, and stem cell in response to progastrin in mice and HER-293 cells. Gastroenterology 140: 583-595, 2011.

7. Xu J, Qian J, Xie X, et al: High density lipoprotein cholesterol promotes the proliferation of bone-derived mesenchymal stem cells via binding scavenger receptor-B type I and activation of PI3K/Akt, MAPK/ERK1/2 pathwys. Mol Cell Biochem 371: 55-64, 2012.

8. Skeen JE, Bhaskar PT, Chen CC, et al: Akt deficiency impairs normal cell proliferation and suppresses oncogenesis in a p53-independent and mTORC1-dependent manner. Cancer Cell 10: 269-280, 2006.

9. Priore R, Dailey L and Basilico C: Downregulation of Akt activity contributes to the growth arrest induced by FGF in chondrocytes. J Cell Physiol 207: 800-808, 2006.

10. Zhang F, Jing S, Ren T, et al: MicroRNA-10b promotes migration of mouse bone marrow-derived mesenchymal stem cells and downregulates E-cadherin expression. Mol Med Rep 8: 1084-1088, 2013

11. Chen BY, Wang X, Chen LW and Luo ZJ: Molecular targeting regulation of proliferation and differentiation of bone marrow-derived mesenchymal stem cells or mesenchymal stromal cells. Curr Drug Targets 13: 561-571, 2012.

12. Wang Y, Chen X,Zhu W, et al: Growth inhibition of mesenchymal stem cells by aspirin: involvement of the WNT/beta-catenin signal pathway. Clin Exp Pharmacol Physiol 33: 696-701, 2006.

13. Hoffman MD and Brnoit DS: Agonism of Wnt- $\beta$-catenin signaling promotes mesenchymal stem cell (MSC) expansion. J Tissue Eng Regen Med: Apr 1, 2013 (Epub ahead of print).

14. Foo C, Frey S, Yang HH, et al: Downregulation of beta-catenin and transdifferentiation of human osteoblast to adipocytes under estrogen deficiency. Gynecol Endocrinol 23: 535-540.

15. Zhang F, Hong Y, Liang W, et al: Co-culture with Sertoli cells promotes proliferation and migration of umbilical cord mesenchyaml stem cells. Biochem Biophys Res Commun 427: 86-90, 2012.

16. Wang RC, Wei Y, An Z, et al: Akt-mediated regulation of autophagy and tumorigenesis through Beclin 1 phosphorylation. Science 338: 956-959, 2012.

17. Xu J, Qian J, Xie X, et al: High density lipoprotein cholesterol promotes the proliferation of bone-derived mesenchymal stem cells via binding scavenger receptor-B type I and activation of PI3K/Akt, MAPK/ERK1/2 pathwys. Mol Cell Biochem 371: 55-64, 2012.

18. Zhang J, Shemezis JR, McQuinn ER, et al: Akt activation by $\mathrm{N}$-cadherin regulates beta-catenin signaling and neuronal differentiation during cortical development. Neural Dev 8: 7, 2013.

19. Wang Z, Havasi A, Gall JM, et al: Beta-catenin promotes survival of renal epithelial cells by inhibiting Bax. J Am Soc Nephrol 20: 1919-1928, 2009.

20. Dihlmann S, Kloor M, Fallsehr C, et al: Regulation of AKT1 expression by beta-catenin/Tcf/Lef signaling in colorectal cancer cells. Carcinogenesis 26: 1503-1512, 2005.

21. Zhang Y, Yu J, Shi C, et al: Regulatory effect of $\beta$-catenin on proliferation of hair follicle stem cells involves PI3K/Akt pathway. J Appl Biomed 11: 131-141, 2013. 